\title{
Rare malignant insulinoma with multiple liver metastases derived from ectopic pancreas: 3-year follow-up and literature review
}

This article was published in the following Dove Press journal:

OncoTargets and Therapy

\author{
Qing Liu' \\ Jianghui Duan ${ }^{2}$ \\ Yumin Zheng ${ }^{3}$ \\ Jie Luo ${ }^{4}$ \\ Xiaopin $\mathrm{Ca}^{5}$ \\ Huangying Tan' \\ 'Department of Integrative Oncology, \\ China-Japan Friendship Hospital, \\ Beijing, China; ${ }^{2}$ Department of \\ Diagnostic Radiology, China-Japan \\ Friendship Hospital, Beijing, China; \\ ${ }^{3}$ Department of Nuclear Medicine, \\ China-Japan Friendship Hospital, \\ Beijing, China; ${ }^{4}$ Department of \\ Pathology, China-Japan Friendship \\ Hospital, Beijing, China; ${ }^{5}$ Department \\ of Endocrinology, China-Japan \\ Friendship Hospital, Beijing, China
}

Correspondence: Huangying Tan Department of Integrative Oncology, China-Japan Friendship Hospital, No. 2 Yinghua Street, Chaoyang District, Beijing 100029, China

Tel +86 1053236555

Fax +86 I0 53236555

Email tanhuangying@263.net

\begin{abstract}
Here, we report the diagnosis and treatment of a very rare case of malignant insulinoma derived from ectopic pancreas. A middle-aged woman presented with a 6-year history of recurrent hypoglycemia with multiple lesions in liver. Admission workup revealed elevated serum insulin and C-peptide, as well as multiple lesions in the liver (largest being $4.3 \mathrm{~cm}$ ), enlarged lymph nodes around the pancreas, and a lesion (of $3.5 \mathrm{~cm}$ ) at the proximal jejunum, as shown by contrast computed tomography (CT). Liver biopsy showed the lesions to be well-differentiated neuroendocrine tumors, grade G1. ${ }^{68} \mathrm{Gallium}-\mathrm{exendin}-4$ positron emission tomography/CT confirmed the origin as the lesion located at the jejunum. The combination treatment of everolimus plus long-acting octreotide relieved symptoms and achieved a partial tumor response. Maintenance treatment of the somatostatin analog (ie, octreotide) alone was administered. Three years of follow-up, up to the writing of this report, showed good survival, with the patient remaining asymptomatic and euglycemic without disease progression. This case shows that ${ }^{68}$ Gallium-exendin-4 positron emission tomography/CT is useful for locating insulinoma, especially for insulinoma derived from ectopic pancreas, and that everolimus plus octreotide with maintenance somatostatin analog alone is an effective drug strategy for treating inoperable malignant insulinoma.
\end{abstract}

Keywords: malignant insulinoma, ectopic pancreas, octreotide, everolimus, ${ }^{68} \mathrm{Gallium}$-exendin-4 positron emission tomography/computed tomography, ${ }^{68} \mathrm{Ga}$-exendin-4 PET/CT

\section{Introduction}

Insulinomas are a rare tumor type, with an annual incidence of $\sim$ four cases per million persons; moreover, nearly $10 \%$ of insulinomas are malignant. ${ }^{1}$ Correspondingly, case reports of malignant insulinoma with distant metastases are scarce in the literature, and those of insulinoma with multiple liver metastases arising from ectopic pancreas are extremely rare.

Ectopic pancreas, also referred to as heterotopic pancreas, is an anatomical abnormality that represents growth of pancreatic tissue outside of the pancreas, with no anatomic or vascular connection. Incidence of ectopic pancreas was about $0.5 \%-15 \%$, and predominantly distributed in the gastric, duodenal, or jejunal mucosa. ${ }^{2}$

Herein we report a rare case of malignant insulinoma derived from ectopic pancreas. Diagnosis was made by means of a novel application of ${ }^{68} \mathrm{Gallium}-\mathrm{exendin}-4$ positron emission tomography/computed tomography $\left({ }^{68} \mathrm{Ga}\right.$-exendin-4 PET/CT). Partial tumor response was achieved with everolimus plus octreotide, and 3-year maintenance with somatostatin analog (SSA) alone was successful. 


\section{Case report}

In April 2014, a 31-year-old woman presented to our hospital with preexisting diagnosis of hepatic hemangioma (made 6 years earlier). Her medical history provided the following clinical course: ultrasound detection of a lesion $(1.7 \times 1.3 \mathrm{~cm})$ in the posterior right lobe of the liver in 2008 and consistent detection of increasing amounts and growth of lesions yearly, with the largest mass measured by ultrasound being $4.3 \times 3.0 \mathrm{~cm}$. In 2012, the patient developed sporadic episodes of confusion, blurred vision, trembling hands, palpitations, and sweating, which resolved spontaneously and usually within 10-15 min. Sequential consultations with a neurologist and psychiatrist led to psychological diagnosis of hysteria or conversion disorder. The symptoms continued to occur and with increased frequency, appearing to correspond with physical activity and to be relieved following food intake. The patient developed a preference for sweet taste and her weight increased by $20 \mathrm{~kg}$. In March 2014, the patient underwent a blood glucose test (fingertip prick) and was found to be hypoglycemic $(2.52 \mathrm{mmol} / \mathrm{L}$; normal range: $3.6-6.1 \mathrm{mmol} / \mathrm{L}$ ), prompting her presentation to our hospital. She did not have any family history of endocrine tumors or diseases.

We performed a complete physical examination upon her admission, and general findings included height of $165 \mathrm{~cm}$, weight of $93 \mathrm{~kg}$, and no palpable lymph nodes in the neck, axilla, or inguinal region. Abdominal examination produced no remarkable findings. Serology testing showed fasting serum glucose $3.4 \mathrm{mmol} / \mathrm{L}$ (normal range: $3.6-6.1 \mathrm{mmol} / \mathrm{mL}$ ), meanwhile serum insulin of $193.3 \mu \mathrm{IU} / \mathrm{mL}$ (normal range: 2.6-24.9 $\mu \mathrm{IU} / \mathrm{mL}$ ) and serum C-peptide of $9.6 \mathrm{ng} / \mathrm{mL}$ (normal range: $1.1-4.4 \mathrm{ng} / \mathrm{mL}$ ), chromogranin A (CgA) of $19.71 \mathrm{ng} / \mathrm{mL}$ (normal: $<100 \mathrm{ng} / \mathrm{mL}$ ), and gastrin of $51.6 \mathrm{pg} / \mathrm{mL}$ (normal: $<100 \mathrm{ng} / \mathrm{mL}$ ); the lowest postprandial blood glucose level was $1.8 \mathrm{mmol} / \mathrm{L}$. Abdomen contrastenhanced CT imaging showed multiple lesions in the liver (largest being $4.3 \times 3.5 \mathrm{~cm}$ ) and one enhanced lesion $(3.5 \mathrm{~cm})$ located at the proximal jejunum; all lesions were intensified by contrast, and the pancreas appeared normal (Figure 1D1 and D3 images).

The patient then underwent liver biopsy. Histological analysis indicated the lesions to be well differentiated and of neuroendocrine tumor (NET) type, grade G1. Immunohistochemical analysis showed the lesions to be $\mathrm{CgA}(+)$, Syn(+), CD56(+), CK8/18(+), Ki-67(+2\%), and insulin focal positive (Figures 2-5). Octreoscan showed high expression of somatostatin receptor (SSTR) in the jejunum area, liver tumors, and mesenteric lymph nodes, but no expression of SSTR was detected in any portion of the pancreas (Figure 6). ${ }^{68} \mathrm{Ga}$-exendin-4 PET/CT confirmed the lesion located at the jejunum section, below the pancreas body, where glucagonlike peptide-1 receptor (GLP-1R) expression was detected and the most hypercaptant area was located (standard uptake value [SUV], 21.7); these findings supported the
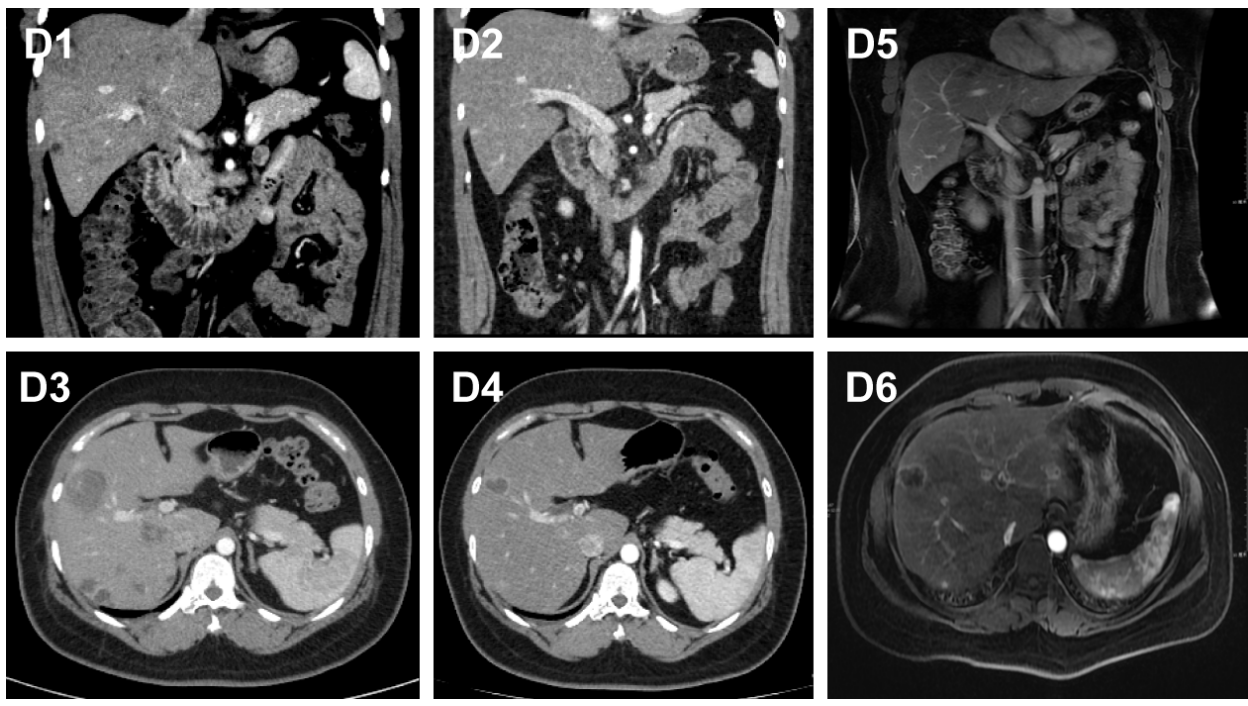

Figure I Contrast-enhanced CT scan of the abdomen taken at baseline (June 20I4) and contrast-enhanced MRI scan of the abdomen taken in June 2017.

Notes: Multiple circular lesions that are evidently strengthened during the arterial period are shown in the liver (image D3). The largest lesion was located in the right lobe S5 $(4.3 \times 3.5 \mathrm{~cm})$. All lesions were considered as metastatic tumors. One enhanced lesion $(3.5 \mathrm{~cm})$ was located at the proximal jejunum (image DI). One year after medical treatment, the liver metastases (image D4) and jejunum lesion (image D2) had reduced and showed decreased density. The largest lesion is located in the right lobe S5 (image D6) and the enhanced lesion is located at the proximal jejunum (image D5).

Abbreviations: $\mathrm{CT}$, computed tomography; MRI, magnetic resonance imaging. 


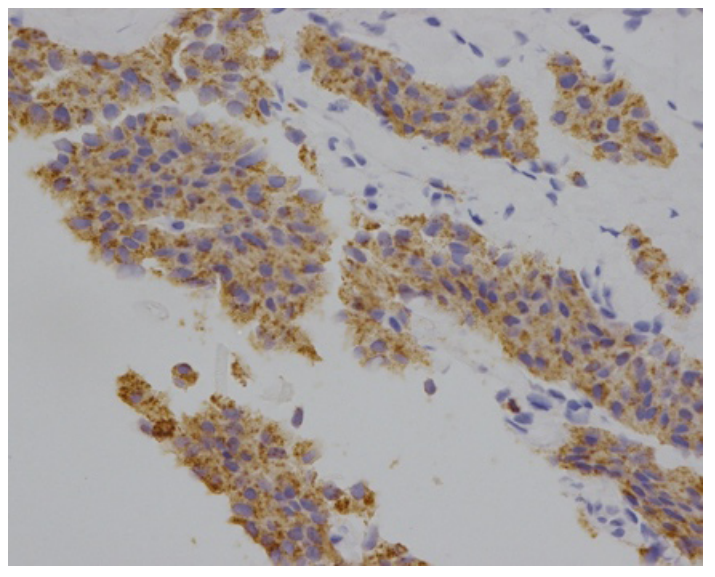

Figure 2 Chromogranin A immunohistochemistry of liver biopsy showing positive staining (10×40 magnification).

lesion to be of NET origin, located outside of the pancreas (Figure 7).

The diagnosis of malignant insulinoma derived from ectopic pancreas was made. This patient was highly suspected of having multiple endocrine neoplasia type 1 (MEN-1). Magnetic resonance imaging (MRI) of the patient's head showed Rathke's cyst within the pituitary gland $(8.6 \times 5.6 \mathrm{~mm}$, as shown in Figure 8). Ultrasound of the parathyroid showed mild hyperplasia, which was accompanied by a slightly elevated serum level of parathyroid hormone (PTH; $104.2 \mathrm{pg} / \mathrm{mL}$, normal range: $12-88 \mathrm{pg} / \mathrm{mL}$ ). Serum and urine levels of growth hormone and calcium were normal, and no symptoms related to the Rathke's cyst were observed.

The patient's multiple metastases precluded surgical therapy to resect all of the insulin-secreting tumors. Thus, in order to control the patient's symptoms and suppress tumor growth, we prescribed everolimus ( $10 \mathrm{mg}$ po $\mathrm{qd}$ ) plus long-acting SSA octreotide (20 mg i.m. Q21d; Sandostatin

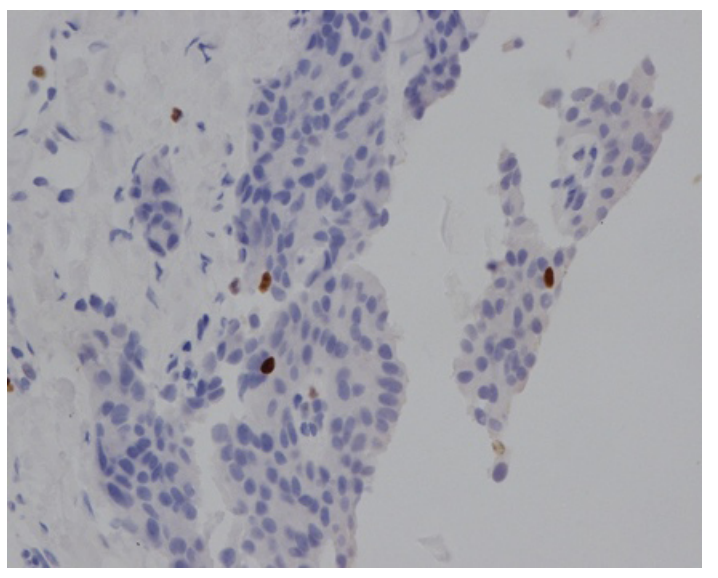

Figure $3 \mathrm{Ki}-67$ (+2\%) of liver biopsy showing positive staining ( $10 \times 40$ magnification).

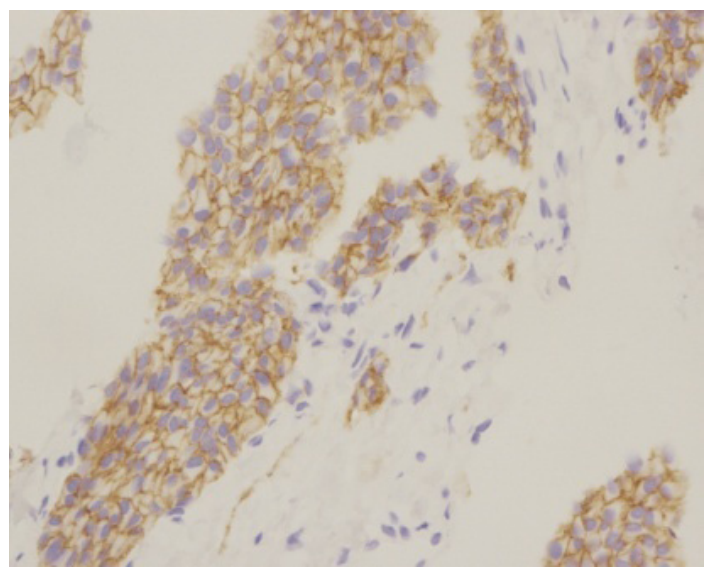

Figure 4 CD56 immunohistochemistry of liver biopsy showing positive staining ( $10 \times 40$ magnification).

LAR, Novartis) starting from June 2014. One week after the treatment initiation, hypoglycemia did not recur. Over the next 4 months of treatment, the largest lesion shrank to $2.2 \times 2.1 \mathrm{~cm}$, the amount of lesions reduced, and the jejunum lesion shrank to $1.8 \mathrm{~cm}$, showing an overall partial tumor response according to Response Evaluation Criteria In Solid Tumors 1.1 criteria.

During the treatment course, the patient developed oral mucositis (grade 2), rashes, and recurrent respiratory infection (grade 2) related to the everolimus administration; these issues lessened upon gradual decrease of the everolimus dose (from 10 to $5 \mathrm{mg} \mathrm{qd}$, and then to $5 \mathrm{mg}$ orally every other day). Since the tumor remained stable during the dosage modification and the patient had experienced so many adverse events related to the drug, the everolimus was stopped in March 2015, from which time that patient was continued on the SSA alone as maintenance treatment. Till the date of this report, the SSA treatment has continued

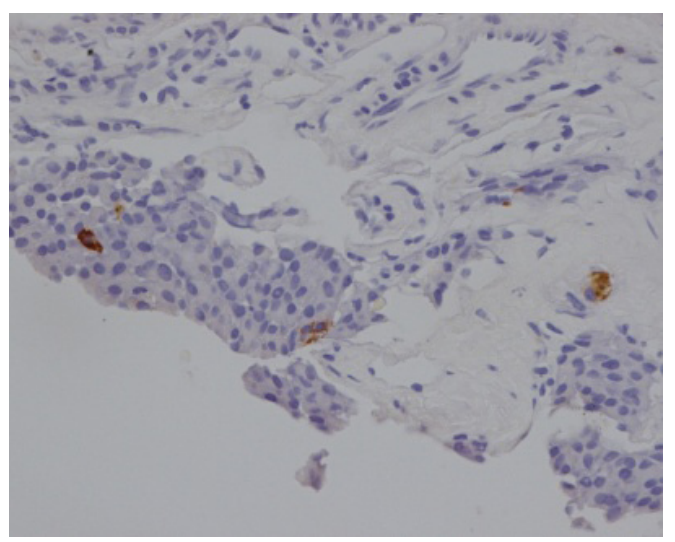

Figure 5 Insulin immunohistochemistry of liver biopsy showing focal positive staining (10×40 magnification) 

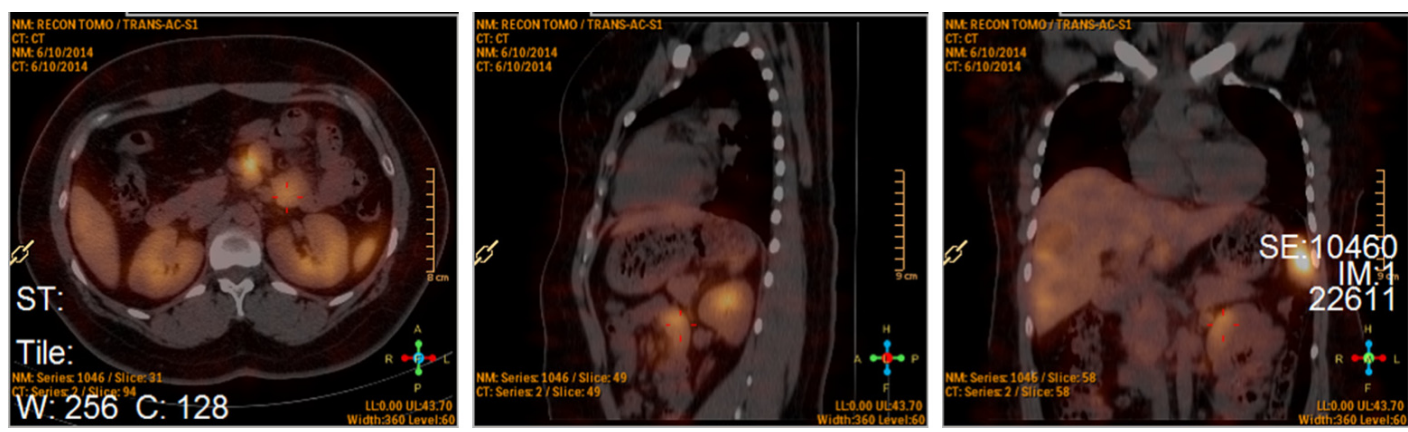

Figure 6 Somatostatin receptor scintigraphy (octreoscan) showed octreotide uptake in liver lesions and ectopic pancreatic tumor located at the area of the jejunum.

uninterrupted (for 3 years now), and no decision has been made on its cessation.

The patient provided written informed consent to authorize use and disclosure of her protected health information for publication.

\section{Follow-up}

The patient attended regular follow-up clinic visits every 2-3 months for the past 3 years; at the time of the writing of this report, the latest follow-up visit had occurred in June 2017. The patient has remained euglycemic and free of symptoms. Her serum level of PTH remained normal, being $86.3 \mathrm{pg} / \mathrm{mL}$ (normal range: $12-88 \mathrm{pg} / \mathrm{mL}$ ) at the latest follow-up visit. Ultimately, the patient tolerated the treatment with SSA alone well, without incidence of side effects and with partial tumor response. The latest images taken by enhanced MRI in June 2017 (Figure 1D5 and D6) shows the partial response of the tumor located in liver and jejunum compared to base line status.

\section{Discussion}

The remarkable increase in incidence of NET over the last few decades has been attributed to improved pathological and diagnostic techniques. According to findings from the Surveillance, Epidemiology and End Results program the pancreatic (p)NET is rare, ${ }^{3}$ with an annual incidence of $1-2$ per 100,000 persons. Insulinomas are the most common functioning pNET, and usually develop as single, small (usually $<2 \mathrm{~cm}$ diameter), benign, and sporadic tumors derived from islet beta cells of the pancreas. ${ }^{4,5}$ The malignant form of insulinoma accounts for $8 \%-10 \%$ of all insulinomas and that derived from ectopic pancreas is extremely rare. As such, the definite incidence of malignant insulinoma derived from ectopic pancreas remains unknown, and estimates are based on the documented cases, which have been presented mainly as case reports in the literature.

The key management steps for insulinoma are as follows. 1) Establishing the diagnosis, including locating the tumor
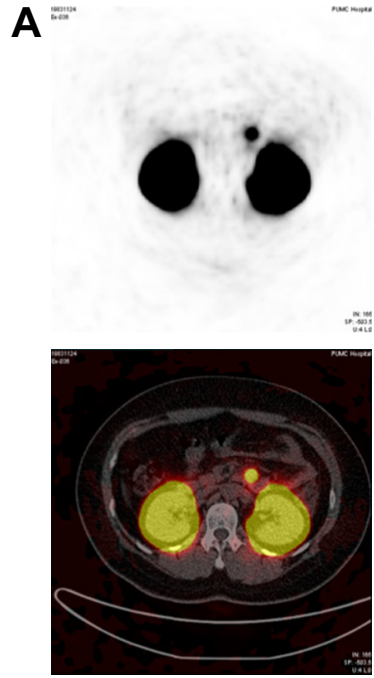
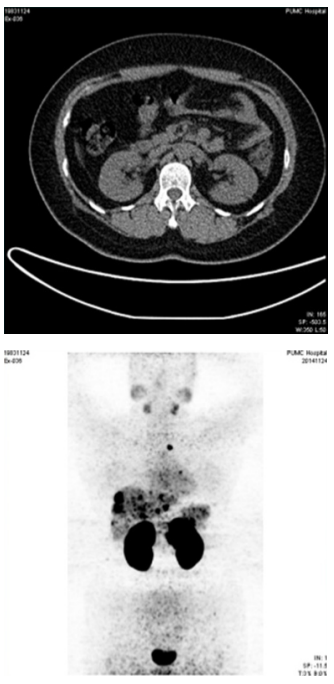

B
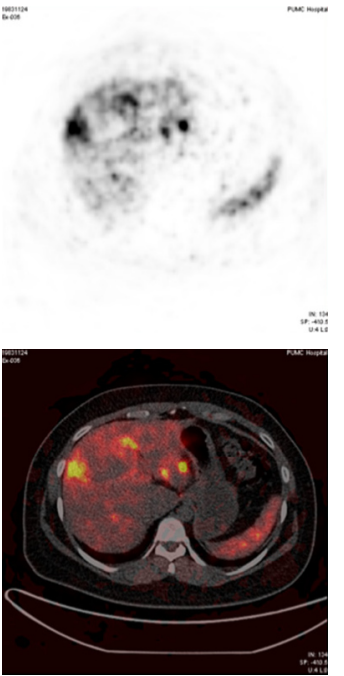

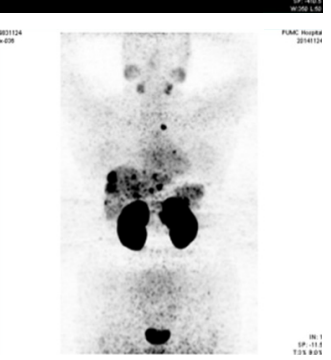

Figure $7{ }^{68} \mathrm{Ga}$-exendin-4 PET/CT.

Notes: (A) There are no definite high expressions of GLP-IR in the entire pancreas, and the lesion was located at the proximal jejunum, below the body of pancreas, where expression of GLP-IR was most hypercaptant (SUV 2I.7). (B) GLP-IR was also expressed in the multiple liver lesions (SUV 2.0-12.5).

Abbreviations: ${ }^{68} \mathrm{Ga}$-exendin-4 PET/CT, ${ }^{68} \mathrm{Gallium}-\mathrm{exendin}-4$ positron emission tomography/computed tomography; GLP-IR, glucagon-like peptide-I receptor; SUV, standard uptake value. 


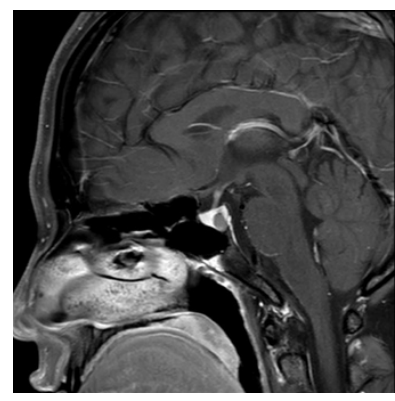

Figure $8 \mathrm{~A}$ nodule between the hypophysis and the posterior lobe was detected by MRI.

Note: The cyst is shown in the sagittal position in the enhanced TI weighted image and was considered as a Rathke's cyst $(8.6 \times 5.6 \mathrm{~mm})$.

Abbreviation: MRI, magnetic resonance imaging.

origin and determining the extent of the tumor by means of localization modalities; this is the most challenging step and requires a multidisciplinary approach. 2) Determining whether an inherited disorder is also present. 3) Designing and implementing a therapeutic strategy that will control hormone-related symptoms and will include surgical resection of the tumor(s) if possible or other antitumor treatment(s) if unresectable. 4) Long-term follow-up of the patient and monitoring of the disease in case of recurrence. ${ }^{6}$

The diagnosis of insulinoma mainly depends on clinical symptoms and results of laboratory testing and imaging examination - the typical clinical manifestations of the Whipple triad. The fasting test also helps to make the diagnoses. The patient reported herein presented with symptomatic hypoglycemic attack, and laboratory tests showed a hyperinsulinemic state during the hypoglycemia attack. Enhanced MRI and CT showed multiple lesions in the liver (metastatic tumors) and a single enhanced lesion located at the proximal jejunum, with a totally normal pancreas. In routine practice, once a biochemical diagnosis of an insulinoma is established, localization diagnosis is performed. The localization of this patient's insulinoma origin was challenging because there was no tumor found in the pancreas. We, therefore, considered the possibility of ectopic pancreas.

Like malignant insulinomas, ectopic pancreas is uncommon, reported to occur in only $0.5 \%-15 \%$ of the population worldwide and with an autopsy incidence of only $0.29 \%-0.71 \%$ in China. ${ }^{7}$ An anatomical abnormality, cases of the ectopic tissue growth have shown predominance for the stomach, duodenum, and jejunum. With the progress of nuclear medicine technologies, however, there is better chance of detecting cases prior to autopsy. The ${ }^{68} \mathrm{Ga}$-exendin- 4 $\mathrm{PET} / \mathrm{CT}$, for example, has already been demonstrated as highly effective for localizing insulinomas.
As we know, NETs are characterized by the presence of SSTRs on their cell surface and could be detected by multiple imaging modalities including SSTR scintigraphy and ${ }^{68} \mathrm{Ga}-$ labeled PET/CT both of which had proven to be much more useful in NET diagnostics due to several technical and biological advantages such as fast clearance, rapid tissue penetration, and low antigenicity. ${ }^{68} \mathrm{Ga}$-DOTATATE has a high affinity to SSTR 2 and SSTR 5, which expressed in insulinomas up to $80 \%$ of cases. ${ }^{8}$ In a previous study, ${ }^{68} \mathrm{Ga}$-labeled PET showed higher diagnostic efficacy than the $\left({ }^{99} \mathrm{~m}\right) \mathrm{Tc}-\mathrm{HYNIC}-\mathrm{TOC}$ SSTR scintigraphy, with a sensitivity of $97 \%$, a specificity of $92 \%$, and an accuracy of $96 \% .^{9}$ In a recent study, ${ }^{68} \mathrm{Ga}$-DOTATATE PET/CT is a noninvasive imaging modality that identifies most insulinomas $(9 / 10,90 \%)$ compared with other modalities including CT, MRI, endoscopic ultrasound, and selective arterial secretagogue injection. ${ }^{10}$ It was also shown that targeting of the GLP-1R, which is highly expressed in insulinomas, using the specific ligand [Lys40(Ahx-DOTA111In) NH2] exendin-4, is a much more sensitive method for localizing insulinomas with PET/CT especially with high sensitivity for hidden small insulinoma. ${ }^{11}$ In China, the ${ }^{68} \mathrm{Ga}$-exendin-4 PET/CT technology is available only at the Peking Union Medical College Hospital, and it has not been approved for routine use in the detection of NETs. Our patient was afforded the opportunity of participating in a clinical trial for this type of detection and the result confirmed the ectopic malignant insulinoma arising from jejunum, as shown in Figure 7. For verification, SSTR scintigraphy (ie, octreoscan) was performed as well and showed all the lesions, particularly the ectopic pancreas expressed SSTR (Figure 6).

MEN-1 is a rare hereditary disease characterized by the development of tumors or hyperplasia in multiple endocrine tissues, including parathyroid, pancreas, and pituitary. ${ }^{12}$ Caused by mutations in the MEN-1 gene at chromosome $11 q 13$, these cases account for only $4 \%$ of insulinoma patients. When an insulinoma is suspected, it is important to determine whether or not MEN-1 is present. Unfortunately, our patient refused the testing for $M E N-1$ gene mutation, the results of which may otherwise have helped to improve the clinical diagnosis rates for this rare disease. However, discussion by the multidisciplinary team (MDT) in our care center agreed that it is highly suspected that this patient might represented a case of MEN-1. The patient's pituitary Rathke's cyst and parathyroid hyperplasia did not require clinical intervention, and observation in follow-up was deemed adequate; however, the possibility of other tumors appearing over her lifetime should be carefully monitored. The key treatment, therefore, focused on the insulinoma derived from ectopic pancreas. 
The treatment of advanced insulinoma aims to control symptoms and the tumor. Drugs such as SSA, sunitinib, and the mTOR inhibitor everolimus have already been demonstrated and established as effective for treating insulinoma and represent the optimal drug available on the market today. ${ }^{13-15}$ Everolimus is also especially effective at controlling hypoglycemia. In a recent retrospective, multicentric study, ${ }^{14} 12$ patients with metastatic insulinoma and refractory hypoglycemia were treated with everolimus and $>91 \%$ experienced normalization of the hypoglycemia, with a median duration of 6.5 months (range: $1-35$ months) to the first recurrence of symptomatic hypoglycemia.

Besides the effect of glycemic control, everolimus also exerts an antitumor effect, significantly improving the prognosis of $\mathrm{pNET}^{16}$ During the everolimus treatment period, patient tolerance should be carefully monitored. The most common adverse effects included asthenia, anorexia, stomatitis, neutropenia, hypertriglyceridemia, and skin rash. Severe side effects have also been reported (in one study, ${ }^{16}$ in up to one-half of the patients) and include pulmonary and/or cardiac events, and may necessitate discontinuation. Our patient developed neutropenia, stomatitis, skin rash over the scalp and back, and recurrent respiratory infection; because of the partial tumor response that had been achieved and stabilized, and the recurrent side effects, everolimus was discontinued after nearly 9 months of administration. The maintenance treatment was with SSA alone on a monthly administration schedule.

SSA is another drug with demonstrated efficacy in the treatment of well-differentiated pNET, capable of exerting both tumor and symptom control. ${ }^{17}$ Following the World Health Organization's 2010 classification system, our patient was diagnosed with NET G1 through liver biopsy; moreover, this diagnosis came at least 6 years after the first liver lesion had become discernable by imaging examination. In terms of safety, SSA is well tolerated in most patients, with only rare reports of digestive reaction or abnormal glucose metabolism. Long-term SSA therapy, however, carries a risk of cholecystolithiasis; as such, patients under this regimen should be followed by ultrasonography.

Our patient received long-acting octreotide for 3 years, making no complaints of related side effects. Thus, this case highlights the potential of an SSA for effectively treating a well-differentiated pNET. Other antitumor options, including hepatic artery embolization and peptide receptor radionuclide therapy, have been demonstrated as effective in some malignant insulinoma patients and may be considered if the disease progresses. ${ }^{17,18}$
The selection of patients with malignant ectopic insulinomas for surgical treatment should be based on the tumor location and related symptoms. Even for some unresectable tumors, palliative debulking of liver metastases can effectively alleviate symptoms and would be beneficial for improving overall survival. ${ }^{19} \mathrm{~A}$ recent review showed that the combination of long-acting SSA therapy with aggressive cytoreductive surgery can result in long-term survival in some patients with metastatic NETs originating in the gastrointestinal tract. ${ }^{20}$

The surgeons in our MDT discussed how to alleviate the patient's tumor burden and obtain pathological confirmation of the insulinoma derived from ectopic pancreas. The detection of multiple lesions in the right lobe of the liver and abdominal lymph nodes could have been addressed by radiofrequency ablation, which could have also afforded the ability to remove other small liver metastases during the operation. But, after the risks associated with the surgery were explained to the patient, she refused the option.

\section{Conclusion}

We present here a rare case of likely MEN-1 with advanced insulinoma derived from ectopic pancreas that was successfully treated with everolimus and SSA, with the latter alone continued as maintenance therapy. In this case report, we also describe the novel application of ${ }^{68} \mathrm{Ga}$-exendin-4 PET/CT as a nuclear imaging tool to help locate hidden insulinomas, suggesting its value in future clinical applications for such cases. Comprehensive therapy is promising for providing improved prognosis of malignant insulinoma, and appropriate drug therapy will enable the patient to have a good quality of life. Widespread discussions on the timing of debulking surgery and whether operative intervention will provide a better survival rate is still needed and will involve more collective data on long-term follow-up of such cases.

\section{Disclosure}

The authors report no conflicts of interest in this work.

\section{References}

1. Service F, McMahon MM, O’Brien PC, Ballard DJ. Functioning insulinoma-incidence, recurrence and long-time survival: a 60-year study. Mayo Clin Proc. 1991;66:711-719.

2. Jeong HY, Yang HY, Yang HW, Seo SW, Seong JK, Na BK. Adenocarcinoma arising from an ectopic pancreas in the stomach. Endoscopy. 2002;34:1014-1017.

3. Ramage JK, Ahmed A, Ardill J, BaxX N, Breen D, Caplin M. Guidelines for the management of gastroenteropancreatic neuroendocrine (including carcinoid) tumors (NETs). Gut. 2012;61(1):616-632.

4. Abboud B, Boujaoude J. Occult sporadic insulinoma: localization and surgical strategy. World J Gastroenterol. 2008;14:657-665. 
5. Oberg K, Knigge U, Kwekkeboom D, Perren A; ESMO Guidelines Working Group. Neuroendocrine gastro-entero-pancreatic tumors: ESMO Clinical Practice Guidelines for diagnosis, treatment and follow-up. Ann Oncol. 2012; 7:vii124-vii130.

6. Ito T, Igarashi H, Jensen RT. Pancreatic neuroendocrine tumors: clinical features, diagnosis and medical treatment: advances. Best Pract Res Clin Gastroenterol. 2012;26:737-753.

7. Wei R, Wang QB, Chen QH, Liu JS, Zhang B. Upper gastrointestinal tract heterotopic pancreas: findings from $\mathrm{CT}$ and endoscopic imaging with histopathologic correlation. Clin Imaging. 2011;35:353-359.

8. Grażyna Ł, Małgorzata B, Agnieszka FW, Izabella KG, Paweł O, Agata SS. The diagnostic role of $68 \mathrm{Ga}$-DOTATATE PET/CT in the detection of neuroendocrine tumours. Nucl Med Rev. 2011;14(1):16-20.

9. Gabriel M, Decristoforo C, Kendler D, et al. 68Ga-DOTA-Tyr3octreotide PET in neuroendocrine tumors: comparison with somatostatin receptor scintigraphy and CT. J Nucl Med. 2007;48:508-518.

10. Pavel N, Bruna B, Corina M, et al. Localization of insulinoma using ${ }^{68} \mathrm{Ga}$-DOTATATE PET/CT scan. J Clin Endocrinol Metab. 2017; 102(1):195-199.

11. Antwi K, Fani M, Nicolas G, et al. Localization of hidden insulinomas with (68) Ga-DOTA-exendin-4 PET/CT: a pilot study. J Nucl Med. 2015;56(7):1075-1078.

12. Jensen RT, Berna MJ, Bingham MD, Norton JA. Inherited pancreatic endocrine tumor syndromes: advances in molecular pathogenesis, diagnosis, management and controversies. Cancer. 2008;113(Suppl 7): 1807-1843.

13. Jawiarczyk A, Bolanowski M, Syrycka J, et al. Effective therapy of insulinoma by using long-acting somatostatin analogue. A case report and literature review. Exp Clin Endocrinol Diabetes. 2012;120:68-72.
14. Bernard V, Lombard-Bohas C, Taquet MC, et al. Efficacy of everolimus in patients with metastatic insulinoma and refractory hypoglycemia. Eur J Endocrinol. 2013;168:665-674.

15. Chen J, Wang C, Han J, et al. Therapeutic effect of sunitinib malate and its influence on blood glucose concentrations in a patient with metastatic insulinoma. Expert Rev Anticancer Ther. 2013;13:737-743.

16. Yao JC, Shah MH, Ito T, et al. Everolimus for advanced pancreatic neuroendocrine tumors. New Engl J Med. 2011;364:514-523.

17. Falconi M, Eriksson B, Kaltsas G, et al. ENETS consensus guidelines update for the management of patients with functional pancreatic neuroendocrine tumors and non-functional pancreatic neuroendocrine tumors. https://www.ncbi.nlm.nih.gov/pubmed/26742109. Neuroendocrinology. 2016;103(2):153-171.

18. Maiza JC, Vezzosi D, Grunenwald S, et al. Treatment with somatostatin analogs and chemoembolization of liver metastases for severe hypoglycemia in malignant insulinomas. J Endocrinol Invest. 2011; $34: 253-258$.

19. Hüttner FJ, Schneider L, Tarantino I, et al. Palliative resection of the primary tumor in 442 metastasized neuroendocrine tumors of the pancreas: a population-based, propensity score-matched survival analysis. Langenbecks Arch Surg. 2015;400:715-723.

20. Deutsch GB, Lee JH, Bilchick AJ. Long term survival with long acting somatostatin analogues plus aggressive cytoreductive surgery in patients with metastatic neuroendocrine carcinoma. J Am Coll Surg. 2015;221:26-36.
OncoTargets and Therapy

\section{Publish your work in this journal}

OncoTargets and Therapy is an international, peer-reviewed, open access journal focusing on the pathological basis of all cancers, potential targets for therapy and treatment protocols employed to improve the management of cancer patients. The journal also focuses on the impact of management programs and new therapeutic agents and protocols on

\section{Dovepress}

patient perspectives such as quality of life, adherence and satisfaction. The manuscript management system is completely online and includes a very quick and fair peer-review system, which is all easy to use. Visit http://www.dovepress.com/testimonials.php to read real quotes from published authors. 